\title{
HYPERSURFACES IN EUCLIDEAN SPACES WITH FINITE TOTAL CURVATURE
}

\author{
Peng Zhu
}

\begin{abstract}
We discuss complete noncompact hypersurfaces in the Euclidean space $\mathbf{R}^{n+1}$ with finite total curvature. We obtain vanishing result and finiteness theorem for the space of $L^{2}$ harmonic 2-forms. These results are generalized versions of results for $L^{2}$ harmonic 1-forms.
\end{abstract}

\section{Introduction}

Shen and Zhu [10] showed that a complete stable immersed minimal hypersurface $M$ in the Euclidean space $\mathbf{R}^{n+1}$ with finite total curvature is hyperplane. Cheng, Cheung and Zhou [4] proved that a complete weakly stable immersed minimal hypersurface $M$ in $\mathbf{R}^{n+1}$ with finite total curvature is hyperplane. $\mathrm{Fu}$ and $\mathrm{Xu}[6]$ discussed a complete submanifold in $\mathbf{R}^{n+p}$ and obtained the dimension of the space of the $L^{2}$ harmonic 1 -forms on $M$ is finite if $M$ has finite total curvature (i.e., $\|\Phi\|_{L^{n}}<+\infty$ ) and finite total mean curvature (i.e., $\|H\|_{L^{n}}<+\infty$ ). Carron [2] obtained the dimension of the space of all $L^{2}$ harmonic $p$-forms is finite if $M$ has finite total curvature and finite total mean curvature. Cavalcante, Mirandola and Vitório [3] proved that if a complete noncompact submanifold $M^{n}(n \geq 3)$ isometric immersed in $\mathbf{R}^{n+p}$ has finite total curvature, then the dimension of the space of the $L^{2}$ harmonic 1-forms on $M$ is finite. Furthermore, they also proved that there exists a positive constant $\delta(n)$, depending only on $n$, such that if $\|\Phi\|_{L^{n}}<\delta(n)$, then there admits no non-trivial $L^{2}$ harmonic 1 -form on $M$. It was showed in [1] that the space of $L^{2}$ harmonic $p$-forms is related with reduced $L^{2}$ cohomology $H_{2}^{p}(M)$. The author [12] studied the existence of the symplectic structure and $L^{2}$ harmonic 2 -forms on complete manifolds by use of the Bochner formula.

In this paper, we discuss a complete noncompact hypersurface $M^{n}$ in the Euclidean space $\mathbf{R}^{n+1}$ with finite total curvature. We obtain vanishing theorem

2010 Mathematics Subject Classification. 53C20; 53C40.

Key words and phrases. Hypersurfaces in the Euclidean space, Total curvature, $L^{2}$ harmonic 2-forms.

Received November 20, 2014; revised February 16, 2016. 
and finiteness theorem for hypersurfaces in the Euclidean space with finite total curvature as follows:

THEOREM 1.1. Suppose that $M^{n}(n \geq 3)$ is an $n$-dimensional complete noncompact hypersurface isometrically immersed in $\mathbf{R}^{n+1}$. There exists a positive constant $\delta(n)$ depending only on $n$ such that if the total curvature $\|\Phi\|_{L^{n}(M)}$ is less than $\delta(n)$, then there admits no non-trivial $L^{2}$ harmonic 2-form on $M$ and the second space of reduced $L^{2}$ cohomology of $M$ is trivial.

THEOREM 1.2. Let $M^{n}(n \geq 3)$ be an $n$-dimensional complete noncompact hypersurface isometrically immersed in $\mathbf{R}^{n+1}$. If the total curvature is finite, then the dimension of the space of all $L^{2}$ harmonic 2-forms and the dimension of the second space of reduced $L^{2}$ cohomology of $M$ are both finite.

\section{Preliminaries}

We recall several definitions. Let $M^{n}$ be an $n$-dimensional Riemannian manifold. The Hodge operator $*: \bigwedge^{p}(M) \rightarrow \bigwedge^{n-p}(M)$ is defined as follows:

$$
* e^{i_{1}} \wedge \cdots \wedge e^{i_{p}}=\operatorname{sgn} \sigma\left(i_{1}, i_{2}, \ldots, i_{n}\right) e^{i_{p+1}} \wedge \cdots \wedge e^{i_{n}},
$$

where $\sigma\left(i_{1}, i_{2}, \ldots, i_{n}\right)$ denotes a permutation of the set $\left(i_{1}, i_{2}, \ldots, i_{n}\right)$ and $\operatorname{sgn} \sigma$ is the sign of $\sigma$. The operator $d^{*}: \bigwedge^{p}(M) \rightarrow \bigwedge^{p-1}(M)$ is given by

$$
d^{*} \omega=(-1)^{(n p+p+1)} * d * \omega .
$$

The Laplacian operator is defined by

$$
\triangle \omega=-d d^{*} \omega-d^{*} d \omega .
$$

A $p$-form $\omega$ is called $L^{2}$-harmonic if $\triangle \omega=0$ and

$$
\int_{M} \omega \wedge * \omega<+\infty .
$$

We denote $H^{p}\left(L^{2}(M)\right)$ by the space of all $L^{2}$ harmonic $p$-forms on $M$.

Suppose that $x: M^{n} \rightarrow \mathbf{R}^{n+1}$ is an isometric immersion of an $n$-dimensional hypersurface $M$ in an $(n+1)$-dimensional Euclidean space. Let $A$ denote the second fundamental form and $H$ the mean curvature of the immersion $x$. Let

$$
\Phi(X, Y)=A(X, Y)-H\langle X, Y\rangle,
$$

for all vector fields $X$ and $Y$, where $\langle$,$\rangle is the induced metric of M$. We say the immersion $x$ has finite total curvature if

$$
\|\Phi\|_{L^{n}(M)}<+\infty \text {. }
$$

We state several results which will be used later. 
Lemma 2.1 [8]. If $\left(M^{n}, g\right)$ is a Riemannian manifold and $\omega=a_{I} \omega_{I} \in \bigwedge^{p}(M)$, then

$$
\triangle|\omega|^{2}=2\langle\triangle \omega, \omega\rangle+2|\nabla \omega|^{2}+2\langle E(\omega), \omega\rangle
$$

where $E(\omega)=R_{k_{\beta} i_{\beta} j_{\alpha} i_{\alpha}} a_{i_{1} \cdots k_{\beta} \cdots i_{p}} e^{i_{p}} \wedge \cdots \wedge e^{j_{\alpha}} \wedge \cdots \wedge e^{i_{1}}$.

Proposition $2.2[1]$. Let $(M, g)$ is a complete Riemannian manifold, then the space of $L^{2}$ harmonic p-forms $H^{p}\left(L^{2}(M)\right)$ is isomorphic to the p-th space of reduced $L^{2}$ cohomology $H_{2}^{p}(M)$.

Proposition 2.3 [7]. Let $M^{n}(n \geq 3)$ be a complete noncompact hypersurface isometrically immersed in $\mathbf{R}^{n+1}$. Then

$$
\left(\int_{M}|f|^{2 n /(n-2)}\right)^{(n-2) / n} \leq C_{0}\left(\int_{M}|\nabla f|^{2}+n^{2} \int_{M} H^{2} f^{2}\right)
$$

for each $f \in C_{0}^{1}(M)$, where $C_{0}$ depends only on $n$ and $H$ is the mean curvature of $M$ in $\mathbf{R}^{n+1}$.

\section{An inequality for $L^{2}$ harmonic 2-forms}

We initially prove an inequality for $L^{2}$ harmonic 2-forms on hypersurfaces in $\mathbf{R}^{n+1}$. Suppose $\omega \in H^{2}\left(L^{2}(M)\right)$ and $h=|\omega|$.

Proposition 3.1. If $M^{n}(n \geq 3)$ is an $n$-dimensional complete noncompact hypersurface isometrically immersed in $\mathbf{R}^{n+1}$, then

$$
h \triangle h \geq\left\{\begin{array}{l}
|\nabla h|^{2}-|\Phi|^{2} h^{2}+\frac{3}{2} H^{2} h^{2} \quad \text { for } n=3, \\
\frac{1}{n-2}|\nabla h|^{2}-\frac{n-2}{2}|\Phi|^{2} h^{2}+n H^{2} h^{2} \quad \text { for } n \geq 4 .
\end{array}\right.
$$

Proof. Since $\omega \in H^{2}\left(L^{2}(M)\right)$, we get that

$$
\triangle|\omega|^{2}=2|\nabla| \omega||^{2}+2|\omega| \triangle|\omega|
$$

Lemma 2.1 implies that

$$
\triangle|\omega|^{2}=2|\nabla \omega|^{2}+2\langle E(\omega), \omega\rangle .
$$

Combining (3.1) with (3.2), we get that

$$
|\omega| \triangle|\omega|=|\nabla \omega|^{2}-|\nabla| \omega||^{2}+\langle E(\omega), \omega\rangle .
$$


Note that there is the Kato inequality for $L^{2}$ harmonic 2-forms [5, 11]:

$$
|\nabla \omega|^{2} \geq \frac{n-1}{n-2}|\nabla| \omega||^{2}
$$

By (3.3) and (3.4), we get that

$$
|\omega| \triangle|\omega| \geq \frac{1}{n-2}|\nabla| \omega||^{2}+\langle E(\omega), \omega\rangle .
$$

Now, we give the estimate of the term $\langle E(\omega), \omega\rangle$. Let $\omega=a_{i_{1} i_{2}} e^{i_{2}} \wedge e^{i_{1}} \in \bigwedge^{2}(M)$, where $a_{i_{1} i_{2}}=-a_{i_{2} i_{1}}$. By Lemma 2.1, we obtain that

$$
\begin{aligned}
E(\omega)= & R_{k_{1} i_{1} j_{1} i_{1}} a_{k_{1} i_{2}} e^{i_{2}} \wedge e^{j_{1}}+R_{k_{2} i_{2} j_{2} i_{2}} a_{i_{1} k_{2}} e^{j_{2}} \wedge e^{i_{1}} \\
& +R_{k_{2} i_{2} j_{1} i_{1}} a_{i_{1} k_{2}} e^{i_{2}} \wedge e^{j_{1}}+R_{k_{1} i_{1} j_{2} i_{2}} a_{k_{1} i_{2}} e^{j_{2}} \wedge e^{i_{1}} \\
= & \operatorname{Ric}_{k_{1} j_{1}} a_{k_{1} i_{2}} e^{i_{2}} \wedge e^{j_{1}}+\operatorname{Ric}_{k_{2} j_{2}} a_{i_{1} k_{2}} e^{j_{2}} \wedge e^{i_{1}} \\
& +R_{k_{2} i_{2} j_{1} i_{1}} a_{i_{1} k_{2}} e^{i_{2}} \wedge e^{j_{1}}+R_{k_{1} i_{1} j_{2} i_{2}} a_{k_{1} i_{2}} e^{j_{2}} \wedge e^{i_{1}} .
\end{aligned}
$$

So, we have that

$$
\begin{aligned}
\langle E(\omega), \omega\rangle= & \operatorname{Ric}_{k_{1} j_{1}} a_{k_{1} i_{2}} a_{j_{1} i_{2}}+\operatorname{Ric}_{k_{2} j_{2}} a_{i_{1} k_{2}} a_{i_{1} j_{2}} \\
& +R_{k_{2} i_{2} j_{1} i_{1}} a_{i_{1} k_{2}} a_{j_{1} i_{2}}+R_{k_{1} i_{1} j_{2} i_{2}} a_{k_{1} i_{2}} a_{i_{1} j_{2}} .
\end{aligned}
$$

By Gauss equation, we have that

$$
R_{i j k l}=h_{i k} h_{j l}-h_{i l} h_{j k} .
$$

A direct computation shows that

$$
\begin{aligned}
\operatorname{Ric}_{k_{1} j_{1}} & =n H h_{k_{1} j_{1}}-h_{k_{1} i} h_{i j_{1}} \\
\operatorname{Ric}_{k_{2} j_{2}} & =n H h_{k_{2} j_{2}}-h_{k_{2} i} h_{i j_{2}} ; \\
R_{k_{2} i_{2} j_{1} i_{1}} & =h_{k_{2} j_{1}} h_{i_{2} i_{1}}-h_{k_{2} i_{1}} h_{i_{2} j_{1}}
\end{aligned}
$$

and

$$
R_{k_{1} i_{1} j_{2} i_{2}}=h_{k_{1} j_{2}} h_{i_{1} i_{2}}-h_{k_{1} i_{2}} h_{i_{1} j_{2}} .
$$

Since the operator is linear $\langle E(\omega), \omega\rangle$ and zero-th order differential operator, it is sufficient to compute $\langle E(\omega), \omega\rangle$ at a point $p$. We can choose an orthonormal frame $\left\{e_{i}\right\}$ such that

$$
h_{i j}=\lambda_{i} \delta_{i j}
$$

at $p$. Note that

$$
n H=\lambda_{1}+\cdots+\lambda_{n}
$$


By (3.6)-(3.10), we obtain that

$$
\begin{aligned}
\langle E(\omega), \omega\rangle= & \sum n H \lambda_{k_{1}}\left(a_{k_{1} i_{2}}\right)^{2}-\sum \lambda_{k_{1}}^{2}\left(a_{k_{1} i_{2}}\right)^{2} \\
& +\sum n H \lambda_{k_{2}}\left(a_{i_{1} k_{2}}\right)^{2}-\sum \lambda_{k_{2}}^{2}\left(a_{i_{1} k_{2}}\right)^{2} \\
& -\sum \lambda_{k_{2} \lambda_{i_{2}}}\left(a_{k_{2} i_{2}}\right)^{2}-\sum \lambda_{j_{2} \lambda_{i_{2}}}\left(a_{j_{2} i_{2}}\right)^{2} \\
= & 2 \sum_{i \neq j}\left(\left(\lambda_{1}+\cdots+\lambda_{n}\right) \lambda_{i}-\lambda_{i}^{2}-\lambda_{i} \lambda_{j}\right)\left(a_{i j}\right)^{2} .
\end{aligned}
$$

Note that

$$
|A|^{2}=|\Phi|^{2}+n H^{2} .
$$

For $n=3$, we get that

$$
\begin{aligned}
\langle E(\omega), \omega\rangle & =2 \sum_{i \neq j}\left(\left(\lambda_{1}+\lambda_{2}+\lambda_{3}\right) \lambda_{i}-\lambda_{i}^{2}-\lambda_{i} \lambda_{j}\right)\left(a_{i j}\right)^{2} \\
& =\sum_{i \neq j}\left(\left(\lambda_{1}+\lambda_{2}+\lambda_{3}\right)\left(\lambda_{i}+\lambda_{j}\right)-\left(\lambda_{i}^{2}+\lambda_{j}^{2}\right)-2 \lambda_{i} \lambda_{j}\right)\left(a_{i j}\right)^{2} \\
& =\sum_{i \neq j}\left(\frac{1}{2}(3 H)^{2}-\frac{1}{2} \sum_{k=1, k \neq i, j}^{3} \lambda_{k}^{2}-\frac{1}{2}\left(\lambda_{i}+\lambda_{j}\right)^{2}\right)\left(a_{i j}\right)^{2} \\
& \geq \sum_{i \neq j}\left(\frac{1}{2}(3 H)^{2}-\frac{1}{2} \sum_{k=1, k \neq i, j}^{3} \lambda_{k}^{2}-\left(\lambda_{i}^{2}+\lambda_{j}^{2}\right)\right)\left(a_{i j}\right)^{2} \\
& \geq \sum_{i \neq j}\left(\frac{9}{2} H^{2}-|A|^{2}\right)\left(a_{i j}\right)^{2}=\left(\frac{3}{2} H^{2}-|\Phi|^{2}\right)|\omega|^{2} .
\end{aligned}
$$

For $n \geq 4$, we have that

$$
\begin{aligned}
\langle E(\omega), \omega\rangle & =2 \sum_{i \neq j}\left(\left(\lambda_{1}+\cdots+\lambda_{n}\right) \lambda_{i}-\lambda_{i}^{2}-\lambda_{i} \lambda_{j}\right)\left(a_{i j}\right)^{2} \\
& =\sum_{i \neq j}\left(\left(\lambda_{1}+\cdots+\lambda_{n}\right)\left(\lambda_{i}+\lambda_{j}\right)-\left(\lambda_{i}^{2}+\lambda_{j}^{2}\right)-2 \lambda_{i} \lambda_{j}\right)\left(a_{i j}\right)^{2} \\
& =\sum_{i \neq j}\left(\left(\lambda_{1}+\cdots+\hat{\lambda}_{i}+\cdots+\hat{\lambda}_{j}+\cdots+\lambda_{n}\right)\left(\lambda_{i}+\lambda_{j}\right)\right)\left(a_{i j}\right)^{2} \\
& =\sum_{i \neq j}\left(\frac{1}{2}(n H)^{2}-\frac{1}{2}\left(\sum_{k=1, k \neq i, j}^{n} \lambda_{k}\right)^{2}-\frac{1}{2}\left(\lambda_{i}+\lambda_{j}\right)^{2}\right)\left(a_{i j}\right)^{2}
\end{aligned}
$$




$$
\begin{aligned}
& \geq \sum_{i \neq j}\left(\frac{1}{2}(n H)^{2}-\frac{n-2}{2}\left(\sum_{k=1, k \neq i, j}^{n} \lambda_{k}^{2}\right)-\left(\lambda_{i}^{2}+\lambda_{j}^{2}\right)\right)\left(a_{i j}\right)^{2} \\
& \geq \sum_{i \neq j}\left(\frac{1}{2}(n H)^{2}-\frac{n-2}{2}|A|^{2}\right)\left(a_{i j}\right)^{2} \\
& =\left(n H^{2}-\frac{n-2}{2}|\Phi|^{2}\right)|\omega|^{2} .
\end{aligned}
$$

By (3.5), (3.11) and (3.12), we obtain the desired result.

\section{Vanishing theorem on hypersurfaces in $\mathbf{R}^{n+1}$}

In this section, we give the proof of Theorem 1.1. If $\eta$ is a compactly supported piecewise smooth function on $M$, then

$$
\operatorname{div}\left(\eta^{2} h \nabla h\right)=\eta^{2} h \triangle h+\eta^{2}|\nabla h|^{2}+2 \eta h\langle\nabla \eta, \nabla h\rangle .
$$

Integrating by parts on $M$, we have that

$$
\int_{M} \eta^{2} h \triangle h+\int_{M} \eta^{2}|\nabla h|^{2}+2 \int_{M} \eta h\langle\nabla \eta, \nabla h\rangle=0 .
$$

Case I: $n=3$. By Proposition 3.1 and (4.1), we obtain that

$$
-2 \int_{M} \eta h\langle\nabla \eta, \nabla h\rangle-2 \int_{M} \eta^{2}|\nabla h|^{2}+\int_{M}|\Phi|^{2} \eta^{2} h^{2}-\frac{3}{2} \int_{M} H^{2} h^{2} \eta^{2} \geq 0 .
$$

Note that

$$
-2 \int_{M} \eta h\langle\nabla \eta, \nabla h\rangle \leq a_{1} \int_{M} \eta^{2}|\nabla h|^{2}+\frac{1}{a_{1}} \int_{M} h^{2}|\nabla \eta|^{2},
$$

for any positive real number $a_{1}$. Set $\phi_{1}(\eta):=\left(\int_{\text {Supp } \eta}|\Phi|^{3}\right)^{1 / 3}$. Then

$$
\begin{aligned}
\int_{M}|\Phi|^{2} \eta^{2} h^{2} \leq & \left(\int_{\text {Supp } \eta}\left(|\Phi|^{2}\right)^{3 / 2}\right)^{2 / 3} \cdot\left(\int_{M}\left(\eta^{2} h^{2}\right)^{3}\right)^{1 / 3} \\
& =\phi_{1}(\eta)^{2} \cdot\left(\int_{M}(\eta h)^{6}\right)^{1 / 3} \\
\leq & C_{0} \phi_{1}(\eta)^{2} \cdot\left(\int_{M}|\nabla(\eta h)|^{2}+9 \int_{M} H^{2}(\eta h)^{2}\right) \\
\leq & C_{0} \phi_{1}(\eta)^{2} \cdot\left(\left(1+\frac{1}{b_{1}}\right) \int_{M} h^{2}|\nabla \eta|^{2}\right. \\
& \left.\quad+\left(1+b_{1}\right) \int_{M} \eta^{2}|\nabla h|^{2}+9 \int_{M} H^{2}(\eta h)^{2}\right)
\end{aligned}
$$


for any positive real number $b_{1}$, where the second inequality holds because of Proposition 2.3. By (4.2)-(4.4), we obtain that

$$
\mathscr{A}_{1} \int_{M} \eta^{2}|\nabla h|^{2}+\mathscr{B}_{1} \int_{M} H^{2} \eta^{2} h^{2} \leq \mathscr{C}_{1} \int_{M} h^{2}|\nabla \eta|^{2}
$$

where

$$
\begin{aligned}
& \mathscr{A}_{1}:=\left(2-C_{0} \phi_{1}(\eta)^{2}\right)-\left(a_{1}+b_{1} C_{0} \phi_{1}(\eta)^{2}\right), \\
& \mathscr{B}_{1}:=\frac{3}{2}-9 C_{0} \phi_{1}(\eta)^{2}
\end{aligned}
$$

and

$$
\mathscr{C}_{1}:=\frac{1}{a_{1}}+C_{0} \phi_{1}(\eta)^{2}\left(1+\frac{1}{b_{1}}\right)
$$

Since the total curvature $\|\Phi\|_{L^{3}(M)}$ is less than $\delta(3)=\sqrt{\frac{1}{6 C_{0}}}, \mathscr{B}_{1}$ and $\mathscr{C}_{1}$ are positive. Choose $a_{1}$ and $b_{1}$ small enough such that $\mathscr{A}_{1}$ is positive. Suppose $B_{r}$ is a geodesic ball of radius $r$ on $M$ centered at a fixed point $p_{0}$. Choose $\eta \in C_{0}^{\infty}(M)$ such that

$$
\left\{\begin{array}{l}
0 \leq \eta \leq 1 \\
\eta \equiv 1 \quad \text { on } B\left(\frac{r}{2}\right) \\
\eta \equiv 0 \quad \text { on } M \backslash B(r), \\
|\nabla \eta| \leq \frac{2}{r}
\end{array}\right.
$$

So (4.5) reduces to

$$
\mathscr{A}_{1} \int_{M} \eta^{2}|\nabla h|^{2}+\mathscr{B}_{1} \int_{M} H^{2} \eta^{2} h^{2} \leq \frac{4 \mathscr{C}_{1}}{r} \int_{M} h^{2} .
$$

Since $\int_{M} h^{2}$ is finite, taking $r \rightarrow+\infty$, we obtain that $h$ is constant and $H^{2} h^{2}=0$. If $h \neq 0$, then $H=0$. Hence, $M$ has infinite volume, contracting the finiteness of $\int_{M} h^{2}$. Therefore, $h=0$.

CASE II: $n \geq 4$. By Proposition 3.1 and (4.1), we get that

$$
\begin{aligned}
-2 \int_{M} \eta h\langle\nabla \eta, \nabla h\rangle-\frac{n-1}{n-2} \int_{M} \eta^{2}|\nabla h|^{2} \\
+\frac{n-2}{2} \int_{M}|\Phi|^{2} \eta^{2} h^{2}-n \int_{M} H^{2} h^{2} \eta^{2} \geq 0 .
\end{aligned}
$$


Note that

$$
-2 \int_{M} \eta h\langle\nabla \eta, \nabla h\rangle \leq a_{2} \int_{M} \eta^{2}|\nabla h|^{2}+\frac{1}{a_{2}} \int_{M} h^{2}|\nabla \eta|^{2}
$$

for any positive real number $a_{2}$. We set $\phi_{2}(\eta):=\left(\int_{\operatorname{Supp} \eta}|\Phi|^{n}\right)^{1 / n}$ and get

$$
\begin{aligned}
\int_{M}|\Phi|^{2} \eta^{2} h^{2} \leq & \left(\int_{\text {Supp } \eta}\left(|\Phi|^{2}\right)^{n / 2}\right)^{2 / n} \cdot\left(\int_{M}\left(\eta^{2} h^{2}\right)^{n /(n-2)}\right)^{(n-2) / n} \\
& =\phi_{2}(\eta)^{2} \cdot\left(\int_{M}(\eta h)^{2 n /(n-2)}\right)^{(n-2) / n} \\
\leq & C_{0} \phi_{2}(\eta)^{2} \cdot\left(\int_{M}|\nabla(\eta h)|^{2}+n^{2} \int_{M} H^{2}(\eta h)^{2}\right) \\
\leq & C_{0} \phi_{2}(\eta)^{2} \cdot\left(\int_{M}\left(1+\frac{1}{b_{2}}\right) h^{2}|\nabla \eta|^{2}\right. \\
& \left.\quad+\left(1+b_{2}\right) \eta^{2}|\nabla h|^{2}+n^{2} \int_{M} H^{2}(\eta h)^{2}\right)
\end{aligned}
$$

for any positive real number $b_{2}$, where the second inequality holds because of Proposition 2.3. By (4.6)-(4.8), we have that

$$
\mathscr{A}_{2} \int_{M} \eta^{2}|\nabla h|^{2}+\mathscr{B}_{2} \int_{M} H^{2} \eta^{2} h^{2} \leq \mathscr{C}_{2} \int_{M} h^{2}|\nabla \eta|^{2}
$$

where

$$
\begin{aligned}
& \mathscr{A}_{2}:=\left(\frac{n-1}{n-2}-\frac{n-2}{2} C_{0} \phi_{2}(\eta)^{2}\right)-\left(a_{2}+\frac{n-2}{2} b_{2} C_{0} \phi_{2}(\eta)^{2}\right), \\
& \mathscr{B}_{2}:=n-\frac{n^{2}(n-2)}{2} C_{0} \phi_{2}(\eta)^{2}
\end{aligned}
$$

and

$$
\mathscr{C}_{2}:=\frac{1}{a_{2}}+\frac{n-2}{2}\left(1+\frac{1}{b_{2}}\right) C_{0} \phi_{2}(\eta)^{2}
$$

Since the total curvature $\|\Phi\|_{L^{n}(M)}$ is less than $\delta(n)=\sqrt{\frac{2}{n(n-2) C_{0}}}$, we have $\mathscr{B}_{2}$ and $\mathscr{C}_{2}$ are positive. Choose $a_{2}$ and $b_{2}$ small enough such that $\mathscr{A}_{2}$ is positive. Let $B_{r}$ be a geodesic ball of radius $r$ on $M$ centered at a fixed point $p_{0}$. Choose $\eta \in C_{0}^{\infty}(M)$ such that 


$$
\left\{\begin{array}{l}
0 \leq \eta \leq 1, \\
\eta \equiv 1 \quad \text { on } B\left(\frac{r}{2}\right), \\
\eta \equiv 0 \quad \text { on } M \backslash B(r), \\
|\nabla \eta| \leq \frac{2}{r}
\end{array}\right.
$$

Let $r \rightarrow+\infty$ in (4.9). We obtain that $h=0$, which is similar to Case I.

Therefore, there admits no nontrivial $L^{2}$-harmonic 2-form on $M$. By Corollary 1.6 in [1], we get that the second space of reduced $L^{2}$ cohomology of $M$ is trivial.

\section{Finiteness theorem on hypersurfaces in $\mathbf{R}^{n+1}$}

In this section, we prove Theorem 1.2.

Suppose $n=3$. By (4.5), we obtain that

$$
\mathscr{A}_{1} \int_{M} \eta^{2}|\nabla h|^{2}+\mathscr{B}_{1} \int_{M} H^{2} \eta^{2} h^{2} \leq \mathscr{C}_{1} \int_{M} h^{2}|\nabla \eta|^{2}
$$

where

$$
\begin{aligned}
& \mathscr{A}_{1}:=\left(2-C_{0} \phi_{1}(\eta)^{2}\right)-\left(a_{1}+b_{1} C_{0} \phi_{1}(\eta)^{2}\right), \\
& \mathscr{B}_{1}:=\frac{3}{2}-9 C_{0} \phi_{1}(\eta)^{2}
\end{aligned}
$$

and

$$
\mathscr{C}_{1}:=\frac{1}{a_{1}}+C_{0} \phi_{1}(\eta)^{2}\left(1+\frac{1}{b_{1}}\right)
$$

Since the total curvature $\|\Phi\|_{L^{3}(M)}$ is finite, we can choose a fixed $r_{0}$ such that

$$
\|\Phi\|_{L^{3}\left(M-B_{r_{0}}\right)}<\delta_{1}=\sqrt{\frac{1}{12 C_{0}}} .
$$

Set

$$
\begin{aligned}
& \tilde{\mathscr{A}}_{1}:=\left(2-C_{0} \delta_{1}^{2}\right)-\left(a_{1}+b_{1} C_{0} \delta_{1}^{2}\right), \\
& \tilde{\mathscr{B}}_{1}:=\frac{3}{2}-9 C_{0} \delta_{1}^{2}
\end{aligned}
$$

and

$$
\tilde{\mathscr{C}}_{1}:=\frac{1}{a_{1}}+C_{0} \delta_{1}^{2}\left(1+\frac{1}{b_{1}}\right) .
$$


HYPERSURFACES IN EUCLIDEAN SPACES WITH FINITE TOTAL CURVATURE 561 Thus,

$$
\tilde{\mathscr{A}}_{1} \int_{M} \eta^{2}|\nabla h|^{2}+\tilde{\mathscr{B}}_{1} \int_{M} H^{2} \eta^{2} h^{2} \leq \tilde{\mathscr{C}}_{1} \int_{M} h^{2}|\nabla \eta|^{2}
$$

for any $\eta \in C_{0}^{\infty}\left(M-B_{r_{0}}\right)$, where $\tilde{\mathscr{A}}_{1}, \tilde{\mathscr{B}}_{1}$ and $\tilde{\mathscr{C}}_{1}$ are positive. By Proposition 2.3 , we have

$$
\begin{aligned}
\frac{1}{C_{0}}\left(\int_{M}(\eta h)^{6}\right)^{1 / 3} \leq & \int_{M}|\nabla(\eta h)|^{2}+9 \int_{M} H^{2}(\eta h)^{2} \\
\leq & \left(1+\frac{1}{c_{1}}\right) \int_{M} h^{2}|\nabla \eta|^{2} \\
& +\left(1+c_{1}\right) \int_{M} \eta^{2}|\nabla h|^{2}+9 \int_{M} H^{2}(\eta h)^{2},
\end{aligned}
$$

for any positive real number $c_{1}$. By (5.2) and (5.3), we have

$$
\begin{aligned}
& \frac{1}{C_{0}}\left(\int_{M}(\eta h)^{6}\right)^{1 / 3} \\
& \quad \leq\left(1+\frac{1}{c_{1}}\right) \int_{M} h^{2}|\nabla \eta|^{2}+\left(1+c_{1}\right) \int_{M} \eta^{2}|\nabla h|^{2}+9 \int_{M} H^{2}(\eta h)^{2} \\
& \quad \leq\left(1+\frac{1}{c_{1}}+\left(1+c_{1}\right) \frac{\tilde{\mathscr{C}}_{1}}{\tilde{\mathscr{A}}_{1}}\right) \int_{M} h^{2}|\nabla \eta|^{2}+\left(9-\left(1+c_{1}\right) \frac{\tilde{\mathscr{B}}_{1}}{\tilde{\mathscr{A}}_{1}}\right) \int_{M} H^{2} \eta^{2} h^{2}
\end{aligned}
$$

Choose a sufficient large $c_{1}$ such that

$$
9-\left(1+c_{1}\right) \frac{\tilde{\mathscr{B}}_{1}}{\tilde{\mathscr{A}}_{1}}<0 .
$$

Then (5.4) implies that

$$
\left(\int_{M}(\eta h)^{6}\right)^{1 / 3} \leq \tilde{A} \int_{M} h^{2}|\nabla \eta|^{2},
$$

for any $\eta \in C_{0}^{\infty}\left(M-B_{r_{0}}\right)$, where $\tilde{A}$ is a positive constant.

Suppose $n \geq 4$. By (4.9), we get

$$
\mathscr{A}_{2} \int_{M} \eta^{2}|\nabla h|^{2}+\mathscr{B}_{2} \int_{M} H^{2} \eta^{2} h^{2} \leq \mathscr{C}_{2} \int_{M} h^{2}|\nabla \eta|^{2}
$$

where

$$
\begin{aligned}
& \mathscr{A}_{2}:=\left(\frac{n-1}{n-2}-\frac{n-2}{2} C_{0} \phi_{2}(\eta)^{2}\right)-\left(a_{2}+\frac{n-2}{2} b_{2} C_{0} \phi_{2}(\eta)^{2}\right), \\
& \mathscr{B}_{2}:=n-\frac{n^{2}(n-2)}{2} C_{0} \phi_{2}(\eta)^{2}
\end{aligned}
$$


and

$$
\mathscr{C}_{2}:=\frac{1}{a_{2}}+\frac{n-2}{2}\left(1+\frac{1}{b_{2}}\right) C_{0} \phi_{2}(\eta)^{2} .
$$

Since the total curvature $\|\Phi\|_{L^{n}(M)}$ is finite, we can choose a fixed $r_{0}$ such that

$$
\begin{gathered}
\|\Phi\|_{L^{n}\left(M-B_{r_{0}}\right)}<\delta_{2}=\sqrt{\frac{1}{n(n-2) C_{0}}} \\
\tilde{\mathscr{A}}_{2}:=\left(\frac{n-1}{n-2}-\frac{n-2}{2} C_{0} \delta_{2}^{2}\right)-\left(a_{2}+\frac{n-2}{2} b_{2} C_{0} \delta_{2}^{2}\right), \\
\tilde{\mathscr{B}}_{2}:=n-\frac{n^{2}(n-2)}{2} C_{0} \delta_{2}^{2}
\end{gathered}
$$

and

$$
\tilde{\mathscr{C}}_{2}:=\frac{1}{a_{2}}+\frac{n-2}{2}\left(1+\frac{1}{b_{2}}\right) C_{0} \delta_{2}^{2} .
$$

Obviously, $\tilde{\mathscr{A}}_{2}, \tilde{\mathscr{B}}_{2}$ and $\tilde{\mathscr{C}}_{2}$ are positive. Thus,

$$
\tilde{\mathscr{A}}_{2} \int_{M} \eta^{2}|\nabla h|^{2}+\tilde{\mathscr{B}}_{2} \int_{M} H^{2} \eta^{2} h^{2} \leq \tilde{\mathscr{C}}_{2} \int_{M} h^{2}|\nabla \eta|^{2},
$$

for any $\eta \in C_{0}^{\infty}\left(M-B_{r_{0}}\right)$. Combining with Proposition 2.3, we get that

$$
\begin{aligned}
& \frac{1}{C_{0}}\left(\int_{M}|\eta h|^{2 n /(n-2)}\right)^{(n-2) / n} \\
& \quad \leq \int_{M}|\nabla(\eta h)|^{2}+n^{2} \int_{M} H^{2}(\eta h)^{2} \\
& \quad \leq\left(1+c_{2}\right) \int_{M} \eta^{2}|\nabla h|^{2}+\left(1+\frac{1}{c_{2}}\right) \int_{M} h^{2}|\nabla \eta|^{2}+n^{2} \int_{M} H^{2} \eta^{2} h^{2}
\end{aligned}
$$

for any positive real number $c_{2}$. By (5.7) and (5.8), we have

$$
\begin{aligned}
\frac{1}{C_{0}}\left(\int_{M}|\eta h|^{2 n /(n-2)}\right)^{(n-2) / n} \leq & \left(1+\frac{1}{c_{2}}+\left(1+c_{2}\right) \frac{\tilde{\mathscr{D}}_{2}}{\tilde{\mathscr{A}}_{2}}\right) \int_{M} h^{2}|\nabla \eta|^{2} \\
& +\left(n^{2}-\left(1+c_{2}\right) \frac{\tilde{\mathscr{B}}_{2}}{\tilde{\mathscr{A}}_{2}}\right) \int_{M} H^{2} \eta^{2} h^{2}
\end{aligned}
$$

We choose a sufficient large $c_{2}$ such that

$$
n^{2}-\left(1+c_{2}\right) \frac{\tilde{\mathscr{B}}_{2}}{\tilde{\mathscr{A}}_{2}}<0 .
$$


Then (5.9) implies that

$$
\left(\int_{M}(\eta h)^{2 n /(n-2)}\right)^{(n-2) / n} \leq \tilde{A} \int_{M} h^{2}|\nabla \eta|^{2},
$$

for any $\eta \in C_{0}^{\infty}\left(M-B_{r_{0}}\right)$, where $\tilde{A}$ is a positive constant depending only on $n$.

Therefore, we show that

$$
\left(\int_{M}(\eta h)^{2 n /(n-2)}\right)^{(n-2) / n} \leq \tilde{A} \int_{M} h^{2}|\nabla \eta|^{2}
$$

for any $\eta \in C_{0}^{\infty}\left(M-B_{r_{0}}\right)$, where $\tilde{A}$ is a positive constant depending only on $n$ $(n \geq 3)$.

Next, the proof follows standard techniques (after inequality (33) in [3]) and uses a Moser iteration argument (lemma 11 in [9]). We include a proof here for the sake of completeness. Choose $r>r_{0}+1$ and $\eta \in C_{0}^{\infty}\left(M-B_{r_{0}}\right)$ such that

$$
\left\{\begin{array}{l}
\eta=0 \quad \text { on } B_{r_{0}} \cup\left(M-B_{2 r}\right), \\
\eta=1 \quad \text { on } B_{r}-B_{r_{0}+1} \\
|\nabla \eta|<\tilde{c} \text { on } B_{r_{0}+1}-B_{r_{0}} \\
|\nabla \eta| \leq \tilde{c} r^{-1} \text { on } B_{2 r}-B_{r}
\end{array}\right.
$$

for some positive constant $\tilde{c}$. Then (5.11) becomes that

$$
\left(\int_{B_{r}-B_{r_{0}+1}} h^{2 n /(n-2)}\right)^{(n-2) / n} \leq \tilde{A} \int_{B_{r_{0}+1}-B_{r_{0}}} h^{2}+\frac{\tilde{A}}{r^{2}} \int_{B_{2 r}-B_{r}} h^{2} .
$$

Letting $r \rightarrow \infty$ and noting that $h \in L^{2}(M)$, we obtain that

$$
\left(\int_{M-B_{r_{0}+1}} h^{2 n /(n-2)}\right)^{(n-2) / n} \leq \tilde{A} \int_{B_{r_{0}+1}-B_{r_{0}}} h^{2}
$$

By Hölder inequality

$$
\int_{B_{r_{0}+2}-B_{r_{0}+1}} h^{2} \leq\left(\int_{B_{r_{0}+2}-B_{r_{0}+1}} h^{2 n /(n-2)}\right)^{(n-2) / n} \cdot\left(\int_{B_{r_{0}+2}-B_{r_{0}+1}} 1^{n / 2}\right)^{2 / n},
$$

we have that

$$
\int_{B_{r_{0}+2}} h^{2} \leq\left(1+\tilde{A} \operatorname{Vol}\left(B_{r_{0}+2}\right)^{2 / n}\right) \int_{B_{r_{0}+1}} h^{2} .
$$

Set

$$
\Psi=\left\{\begin{array}{l}
\left.|| \Phi\right|^{2}-\frac{3}{2} H^{2} \mid, \quad \text { for } n=3 \\
\left.\left|\frac{n-2}{2}\right| \Phi\right|^{2}-n H^{2} \mid, \quad \text { for } n \geq 4
\end{array}\right.
$$


Fix $x \in M$ and take $\tau \in C_{0}^{1}\left(B_{1}(x)\right)$. By Proposition 3.1, we have

$$
h \triangle h \geq \alpha|\nabla h|^{2}-\Psi h^{2},
$$

where

$$
\alpha=\left\{\begin{array}{l}
\frac{1}{2}, \quad \text { for } n=3, \\
\frac{1}{n-2}, \text { for } n \geq 4 .
\end{array}\right.
$$

Then, for $p>2$, there exsits

$$
\int_{M} \tau^{2} h^{p-1} \triangle h \geq \alpha \int_{M} \tau^{2} h^{p-2}|\nabla h|^{2}-\int_{M} \tau^{2} \Psi h^{p} .
$$

That is,

$$
\begin{aligned}
-2 \int_{B_{1}(x)} \tau h^{p-1}\langle\nabla \tau, \nabla h\rangle \geq & (\alpha+(p-1)) \int_{B_{1}(x)} \tau^{2} h^{p-2}|\nabla h|^{2} \\
& -\int_{B_{1}(x)} \tau^{2} \Psi h^{p} .
\end{aligned}
$$

Note that

$$
\begin{aligned}
-2 \tau h^{p-1}\langle\nabla \tau, \nabla h\rangle & =-2\left\langle h^{p / 2} \nabla \tau, \tau h^{p / 2-1} \nabla h\right\rangle \\
& \leq \frac{1}{\alpha} h^{p}|\nabla \tau|^{2}+\alpha \tau^{2} h^{p-2}|\nabla h|^{2} .
\end{aligned}
$$

By (5.14), we have that

$$
(p-1) \int_{B_{1}(x)} \tau^{2} h^{p-2}|\nabla h|^{2} \leq \int_{B_{1}(x)} \Psi \tau^{2} h^{p}+\frac{1}{\alpha} \int_{B_{1}(x)}|\nabla \tau|^{2} h^{p} .
$$

Combining Cauchy-Schwarz inequality with (5.15), we get that

$$
\int_{B_{1}(x)}\left|\nabla\left(\tau h^{p / 2}\right)\right|^{2} \leq \int_{B_{1}(x)} \mathscr{A} \Psi \tau^{2} h^{p}+\mathscr{B}|\nabla \tau|^{2} h^{p},
$$

where $\mathscr{A}=\frac{1}{p-1}\left(\frac{p^{2}}{4}+\frac{p}{2}\right)$ and $\mathscr{B}=\left(1+\frac{p}{2}\right)+\frac{1}{\alpha(p-1)}\left(\frac{p^{2}}{4}+\frac{p}{2}\right)$. Choose $f=\tau h^{p / 2}$ in Proposition 2.3. By (5.16), we obtain that

$$
\left(\int_{B_{1}(x)}\left(\tau h^{p / 2}\right)^{2 n /(n-2)}\right)^{(n-2) / 2} \leq p \mathscr{C} \int_{B_{1}(x)}\left(\tau^{2}+|\nabla \tau|^{2}\right) h^{p},
$$

where $\mathscr{C}$ depends on $n$ and $\sup _{B_{1}(x)} \Psi$. Set $p_{k}=\frac{2 n^{k}}{(n-2)^{k}}$ and $\rho_{k}=\frac{1}{2}+\frac{1}{2^{k+1}}$ for $k=0,1,2, \ldots$ Take a function $\tau_{k} \in C_{0}^{\infty}\left(B_{\rho_{k}(x)}\right)$ satisfying: 


$$
\left\{\begin{array}{l}
0 \leq \tau_{k} \leq 1 \\
\tau_{k}=1 \quad \text { on } B_{\rho_{k+1}}(x) \\
\left|\nabla \tau_{k}\right| \leq 2^{k+3}
\end{array}\right.
$$

Choosing $p=p_{k}$ and $\tau=\tau_{k}$ in (5.17), we obtain that

$$
\left(\int_{B_{\rho_{k+1}}(x)} h^{p_{k+1}}\right)^{1 /\left(p_{k+1}\right)} \leq\left(\mathscr{C} p_{k} 4^{k+4}\right)^{1 / p_{k}}\left(\int_{B_{\rho_{k}}(x)} h^{p_{k}}\right)^{1 / p_{k}} .
$$

By recurrence, we have

$$
\|h\|_{L^{p_{k+1}\left(B_{1 / 2}(x)\right)}} \leq \prod_{i=0}^{k} p_{i}^{1 / p_{i}} 4^{i / p_{i}}\left(\mathscr{C} 4^{4}\right)^{1 / p_{i}}\|h\|_{L^{2}\left(B_{1}(x)\right)} \leq \mathscr{D}\|h\|_{L^{2}\left(B_{1}(x)\right)},
$$

where $\mathscr{D}$ is a positive constant depending only on $n, \operatorname{Vol}\left(B_{r_{0}+2}\right)$ and $\sup _{B_{r_{0}+2}} \Psi$. Letting $k \rightarrow \infty$, we get

$$
\|h\|_{L^{\infty}\left(B_{1 / 2}(x)\right)} \leq \mathscr{D}\|h\|_{L^{2}\left(B_{1}(x)\right)} .
$$

Now, choose $y \in \bar{B}_{r_{0}+1}$ such that $\sup _{B_{r_{0}+1}} h^{2}=h(y)^{2}$. Note that $B_{1}(y) \subset B_{r_{0}+2}$. (5.20) implies that

$$
\sup _{B_{r_{0}+1}} h^{2} \leq \mathscr{D}\|h\|_{L^{2}\left(B_{1}(y)\right)}^{2} \leq \mathscr{D}\|h\|_{L^{2}\left(B_{r_{0}+2}\right)}^{2} .
$$

By (5.13), we have

$$
\sup _{B_{r_{0}+1}} h^{2} \leq \mathscr{F}\|h\|_{L^{2}\left(B_{r_{0}+1}\right)}^{2},
$$

where $\mathscr{F}$ depends only on $n, \operatorname{Vol}\left(B_{r_{0}+2}\right)$ and $\sup _{B_{r_{0}+2}} \Psi$. In order to show the finiteness of the dimension of $H^{2}\left(L^{2}(M)\right)$, it suffices to prove that the dimension of any finite dimensional subspaces of $H^{2}\left(L^{2}(M)\right)$ is bounded above by a fixed constant. Combining (5.22) with Lemma 11 in [9], we show that $\operatorname{dim} H^{2}\left(L^{2}(M)\right)<+\infty$. By Proposition 2.2, we have that the dimension of the second space of reduced $L^{2}$ cohomology of $M$ is finite.

Acknowledgements. This work was partially supported by NSFC Grants 11471145, 11371309 and Qing Lan Project.

\section{REFERENCES}

[1] G. CARron, $L^{2}$ harmonic forms on non compact manifolds, arXiv:0704.3194v1, 2007.

[2] G. Carron, $L^{2}$-cohomologie et ingalits de Sobolev, Math. Ann. 314 (1999), 613-639 (in French).

[ 3 ] M. P. Cavalcante, H. Mirandola and F. Vitório, $L^{2}$-harmonic 1-forms on submanifolds with finite total curvature, J. Geom. Anal. 24 (2014), 205-222.

[4] X. Cheng, L. F. Cheung and D. T. Zhou, The structure of weakly stable constant mean curature hypersurfaces, Tohoku Math. J. 60 (2008), 101-121. 
[ 5 ] D. Cibotaru and P. Zhu, Refined Kato inequalities for harmonic fields on Kähler manifolds, Pacific J. Math. 256 (2012), 51-66.

[6] H. P. Fu AND H. W. XU, Total curvature and $L^{2}$ harmonic 1-forms on complete submanifolds in space forms, Geom. Dedicata 144 (2010), 129-140.

[7] D. Hoffman And J. Spruck, Sobolev and isoperimetric inequalities for Riemannian submanifolds, Comm. Pure Appl. Math. 27 (1974), 715-727.

[8] P. LI, Lecture notes on geometric analysis, Lecture notes series 6, Research Institute of Mathematics and Global Analysis Reseach Center, Seoul National University, Seoul, 1993.

[9] P. LI, On the Sobolev constant and the p-spectrum of a compact Riemannian manifold, Ann. Sci. Éc. Norm. Super. 13 (1980), 451-468.

[10] Y. Shen AND X. H. ZhU, On stable complete minimal hypersurfaces in $\mathbf{R}^{n+1}$, Amer. J. Math. 120 (1998), 103-116.

[11] X. D. WANG, On the $L^{2}$-cohomology of a convex cocompact hyperbolic manifold, Duke Math. J. 115 (2002), 311-327.

[12] P. ZHU, Harmonic two-forms on manifolds with nonnegative isotropic curvature, Ann. Glob. Anal. Geom. 40 (2011), 427-434.

Peng Zhu

School of Mathematics and Physics

JiAngsu University of TeChNOLOGY

Changzhou 213001, Jiangsu

P. R. CHINA

E-mail: zhupeng2004@126.com 